\title{
NLTE solar flare models with stationary velocity fields
}

\author{
T. Nejezchleba \\ Astronomical Institute, Academy of Sciences of the Czech Republic, CZ-25165 Ondřejov, Czech Republic
}

Received July 29; accepted May 27, 1997

\begin{abstract}
A method of calculating NLTE models of a plan-parallel solar flare atmosphere with stationary plasma flows is presented. To solve the radiative transfer equation, equations of statistical equilibrium (ESE) and the stationary momentum equation, we use the multilevel approximate lambda iteration (MALI) approach. The numerical code is based on the method recently developed by Rybicky \& Hummer $(1991,1992)$ and allows to take into account macroscopic velocity fields in observer's frame formulation. The preconditioned ESE, constructed by this method, are finally linearized with respect to level populations and electron densities to treat the the hydrogen ionization balance. The numerical code based on this method is used to compute a grid of NLTE flare models with various velocity fields in order to show the influence of the velocity fields on the $\mathrm{H} \alpha$-line asymmetries.

The analysis of the results lead to conclusions that would improve interpretations of flare line asymmetries: 1) The velocities affect the level populations, 2) The type of asymmetry depends on the changes in the optical depth scales and on the run of the source function in the atmosphere. 3) A monotonous velocity affects only one wing of the line profile. 4) To get the velocity field from an observed profile the bisector method should be modified.
\end{abstract}

Key words: line: formation — line: profiles — radiative transfer — methods: numerical — Sun: flares

\section{Introduction}

The asymmetries of chromospheric line profiles (Balmer series of hydrogen, CaII lines, ...) as well as soft X-ray lines $(\mathrm{Ca}$ XIX, Fe XXV, ...) are related to mass motions in the flare atmosphere. The most extended observational data are available for the $\mathrm{H} \alpha$ line that is regarded as the most important tool for analysis. The observations reveal $\mathrm{H} \alpha$-line asymmetries of various kind. While the red asymmetry is present in most flares, the blue one was detected only in some of them and only at the very onset of the flare

Send offprint requests to: T. Nejezchleba
(Švestka 1976; Wülser 1987). The red asymmetry of $\mathrm{H} \alpha$ appears either as a shift of the peak to longer wavelength (Ichimoto \& Kurokawa 1984) or as a stronger red wing of the line (Švestka 1976). It is observed throughout the impulsive phase of the flare, reaches its maximum before the maximum of the line intensity and is temporally correlated with X-ray radiation (Ichimoto \& Kurokawa 1984; Canfield et al. 1990). The blue asymmetry of $\mathrm{H} \alpha$ is almost every time connected with central reversal (Canfield et al. 1990; Heinzel et al. 1994).

The velocities of the chromospheric plasma responsible for observed asymmetries are usually obtained using bisector technique. So Ichimoto \& Kurokawa (1984) found downward motion velocities between $40-100 \mathrm{~km} \mathrm{~s}^{-1}$ and they report drop from 100 to $40 \mathrm{~km} \mathrm{~s}^{-1}$ within $30 \mathrm{~s}$. Wülser \& Marti (1989) get drop from 90 to $30 \mathrm{~km} \mathrm{~s}^{-1}$ within $12 \mathrm{~s}$. Although the bisector technique is widely used, because of its straightforwardness, from the radiative transfer point of view it cannot be generally applied and as was shown by Heinzel et al. (1994) it may lead to misleading results.

The numerical simulations show that the heating of the chromosphere during a flare produces upward as well as downward motions of the chromospheric plasma (Somov et al. 1982; Fisher et al. 1985; Karlický \& Hénoux 1992). These simulations predict an explosive evaporation with upward velocities up to $500 \mathrm{~km} \mathrm{~s}^{-1}$, accompanied by a chromospheric condensation - a thin dense and cool layer propagating downward with velocity up to $90 \mathrm{~km} \mathrm{~s}^{-1}$. Various observations based on bisector method seem to confirm this result (Zarro et al. 1988; Zarro \& Canfield 1989; Wülser \& Marti 1989; Canfield et al. 1990; Wülser et al. 1993).

Falchi et al. (1992) observed a flare in the $\mathrm{H} \alpha, \mathrm{H} \beta, \mathrm{H} \gamma$, $\mathrm{H} \delta$, CaII $\mathrm{K}$ and $\mathrm{Na} \mathrm{D}_{2}$ lines and their results show that the chromospheric condensation probably has a velocity gradient. From the observation of two flares in the $\mathrm{H} \alpha$, Ca II K, He I D $D_{3}$, Na I $D_{1,2}$ and other metallic lines Shoji \& Kurokawa (1995) conclude that flare emission comes from two layers - one thin, fast-downward-moving and very turbulent, and another stationary and optically thick. 
Fang et al. (1992) analysed red asymmetry of CaII K line for 12 solar flares using semi-empirical NLTE calculations. They found the observed asymmetry can be explained by downward motion above temperature minimum region with velocity $10-30 \mathrm{~km} \mathrm{~s}^{-1}$. Using $\mathrm{H} \alpha$ profiles for two flares observed by Wülser \& Marti (1989) and Graeter (1990) Gan et al. (1993) constructed semi-empirical NLTE models with chromospheric condensation. They showed that the chromospheric condensation is responsible not only for red asymmetry of $\mathrm{H} \alpha$ but also for blue asymmetry with central reversal.

A realistic determination of the velocity structure in the flaring atmosphere is extremely important for better understanding of the flare energetic processes. It is highly desirable to replace the simple bisector method by the NLTE dynamic model calculations and compare the synthetic profiles of a number of various lines with the observed ones.

In this paper we would like to present NLTE simulations of the flare atmosphere with prescribed run of the temperature and the velocity field, in order to show the influence of velocities on emergent hydrogen $\mathrm{H} \alpha$-line intensities. The results are compared with the bisector technique.

The NLTE method we used is described in Sect. 2, the models we performed the calculations for are described in Sect. 3 and in Sect. 4 we discuss the results.

\section{Method}

Some authors used static NLTE models to determine flare atmosphere parameters and then performed the formal solution of the radiative transfer equation with a prescribed run of the velocity to evaluate the emergent intensities. For macroscopic velocities comparable with the thermal velocity this can be a valid simplification. However, one can imagine that this approach must fail for velocities that exceed a certain limit. Thus we need a method that consistently includes the velocities. We will show that dynamic NLTE models based on ALI-approach can be satisfactorily used. Here we briefly summarize the multilevel ALI method of Rybicky \& Hummer (1992) (so-called MALI) and point out the modifications needed for flare modeling.

\section{1. $M A L I$}

The formal solution of radiative transfer equation (RTE) can be written down as the lambda operator acting on the source function, $I_{\mu \nu}=\Lambda_{\mu \nu}\left[S_{\mu \nu}\right]$, where $\mu$ is directional cosine and $\nu$ means frequency. The idea of ALI method is to split the lambda operator in two parts and to take the source function in the second term as known from previous iteration (denoted by $\dagger$ ), i.e.

$$
I_{\mu \nu}=\Lambda_{\mu \nu}^{*}\left[S_{\mu \nu}\right]+\left(\Lambda_{\mu \nu}-\Lambda_{\mu \nu}^{*}\right)\left[S_{\mu \nu}^{\dagger}\right]
$$

By choosing an appropriate approximate lambda operator $\Lambda_{\mu \nu}^{*}$ we obtain desired accelerated lambda iteration scheme.

It was shown that the approximate lambda operator $\Lambda_{\mu \nu}^{*}$ working with an optimal convergence rate is the diagonal part of the true lambda operator (Olson et al. 1986). To construct the diagonal $\Lambda_{\mu \nu}^{*}$ we use the method based on Feautrier variables as proposed by Rybicky \& Hummer (1991). Static Feautrier variables can be used in the continuum radiative transfer equation, because it is not affected by the moving plasma.

$\begin{aligned} u & \equiv \frac{1}{2}[I(\mu, \nu)+I(-\mu, \nu)], \\ v & \equiv \frac{1}{2}[I(\mu, \nu)-I(-\mu, \nu)], \\ \mu \geq 0 & \end{aligned}$

For line radiative transfer in a moving media the Feautrier variables are defined as (Mihalas 1978)

$u \equiv \frac{1}{2}[I(\mu, \Delta \nu)+I(-\mu,-\Delta \nu)]$,
$v \equiv \frac{1}{2}[I(\mu, \Delta \nu)-I(-\mu,-\Delta \nu)]$,

where $\Delta \nu \equiv \nu-\nu_{0}$ is a distance from the line center. The line source function has to satisfy the condition $S(\mu, \Delta \nu)=S(-\mu,-\Delta \nu)$, so these Feautrier variables can be used only for non-overlapping lines.

Instead of the lambda operator we use the psi operator that acts on total emissivity $\eta_{\mu \nu}$

$I_{\mu \nu}=\Psi_{\mu \nu}^{*}\left[\eta_{\mu \nu}\right]+\left(\Psi_{\mu \nu}-\Psi_{\mu \nu}^{*}\right)\left[\eta_{\mu \nu}^{\dagger}\right]$

This choice appears to be the most useful for our problem formulation (for details see Rybicky \& Hummer 1992) and thus the lambda operator will be substituted in final formulas.

\section{2. $E S E$}

The general form of equations of statistical equilibrium is (Mihalas 1978)

$\frac{\partial n_{l}}{\partial t}+\nabla \cdot\left(n_{l} \boldsymbol{v}\right)=\sum_{l^{\prime}} n_{l^{\prime}}\left(C_{l^{\prime} l}+R_{l^{\prime} l}\right)-\sum_{l^{\prime}} n_{l}\left(C_{l l^{\prime}}+R_{l l^{\prime}}\right) .(5)$

$n_{l}$ is the atomic level population of the level $l$ and $C_{l l^{\prime}}$ and $R_{l l^{\prime}}$ are, respectively, the collisional and radiative transition rates from level $l$ to $l^{\prime}$. Levels are ordered according to their energy (i.e. $E_{l}>E_{l^{\prime}}$ for $l>l^{\prime}$ ). In this exploratory work we don't consider the non-thermal collisional rates.

If we assume the relaxation times of collisional and $\mathrm{ra}$ diative processes to be much shorter than hydrodynamic 
ones, the statistical equilibrium is not affected by the hydrodynamics of the plasma. Thus we can write the equations of statistical equilibrium in their standard static form

$\sum_{l^{\prime}} n_{l^{\prime}}\left(C_{l^{\prime} l}+R_{l^{\prime} l}\right)=\sum_{l^{\prime}} n_{l}\left(C_{l l^{\prime}}+R_{l l^{\prime}}\right)$

Using the formulation of Rybicky \& Hummer (1992) which takes into account the angle dependence of the absorption coefficient, we express the radiative rates as

$R_{l l^{\prime}}=\oint \mathrm{d} \Omega \int \frac{\mathrm{d} \nu}{h \nu}\left[U_{l l^{\prime}}(\mu, \nu)+V_{l l^{\prime}}(\mu, \nu) I_{\mu \nu}\right]$.

$I_{\mu \nu}$ is the specific intensity for frequency $\nu$ and directional cosine $\mu$. The quantities $U_{l l^{\prime}}$ and $V_{l l^{\prime}}$ are defined for each transition between levels $l$ and $l^{\prime}$. For line transitions there are given by

$$
\begin{aligned}
U_{l l^{\prime}}(\mu, \nu) & \equiv \frac{h \nu}{4 \pi} A_{l l^{\prime}} \varphi_{l l^{\prime}}(\mu, \nu), l>l^{\prime} \\
& \equiv 0, \quad l<l^{\prime} \\
V_{l l^{\prime}}(\mu, \nu) & \equiv \frac{h \nu}{4 \pi} B_{l l^{\prime}} \varphi_{l l^{\prime}}(\mu, \nu),
\end{aligned}
$$

where $A_{l l^{\prime}}$ and $B_{l l^{\prime}}$ are the Einstein coefficients and $\varphi_{l l^{\prime}}$ is the line profile function. Its dependence on $\mu$ for a vertical macroscopic velocity $v$ takes the form

$\varphi_{l l^{\prime}}(\mu, \nu)=\varphi_{l l^{\prime}}\left(\nu-\frac{\mu v}{c} \nu_{0}\right)$

where $\nu_{0}$ is the line center frequency. by

For continuum transitions the $U_{l l^{\prime}}$ and $V_{l l^{\prime}}$ are given

$$
\begin{array}{rlr}
U_{l l^{\prime}}(\nu) & \equiv n_{\mathrm{e}} \Phi_{l l^{\prime}}(T) \frac{2 h \nu^{3}}{c^{2}} \mathrm{e}^{-h \nu / k T} a_{l l^{\prime}}(\nu), l>l^{\prime} \\
& \equiv 0, & l<l^{\prime} \\
V_{l l^{\prime}}(\nu) & \equiv n_{\mathrm{e}} \Phi_{l l^{\prime}}(T) \mathrm{e}^{-h \nu / k T} a_{l l^{\prime}}(\nu), l>l^{\prime} \\
& \equiv a_{l l^{\prime}}(\nu), & l<l^{\prime},
\end{array}
$$

where $a_{l l^{\prime}}$ is the photo-ionization cross section and $\Phi_{l l^{\prime}}(T)$ is the Saha-Boltzmann function (see Mihalas 1978).

The total emissivity can be expressed as

$$
\begin{aligned}
& \eta_{\mu \nu}=\sum_{l l^{\prime}} n_{l} U_{l l^{\prime}}+\eta_{\mathrm{b}} \\
& \eta_{\mu \nu}^{\dagger}=\sum_{l l^{\prime}} n_{l}^{\dagger} U_{l l^{\prime}}+\eta_{\mathrm{b}}
\end{aligned}
$$

where $\eta_{\mathrm{b}}$ is the background emissivity.

Substituting for $\eta_{\mu \nu}$ in Eq. (4) we obtain the resulting formula for intensity

$$
I_{\mu \nu}=\Psi_{\mu \nu}\left[\eta_{\mu \nu}^{\dagger}\right]+\sum_{l l^{\prime}} \Psi_{\mu \nu}^{*}\left[n_{l} U_{l l^{\prime}}\right]-\sum_{l l^{\prime}} \Psi_{\mu \nu}^{*}\left[n_{l}^{\dagger} U_{l l^{\prime}}\right]
$$

Note that $\eta_{\mathrm{b}}$ has cancelled in the second and third term.

\subsection{Momentum equation}

The momentum equation reduces to the equation of hydrostatic equilibrium in the case of a static atmosphere. Assumption that the time changes of the velocity are small $(\partial \boldsymbol{v} / \partial t \equiv 0)$ leads to the stationary form of the momentum equation. Thus

$p+\rho v^{2}=m g_{\odot}$,

where $m$ is the column mass, $p$ is the total pressure, $\rho$ is the density and $g_{\odot}$ is the surface gravity. The second term on the lefthand side will decrease the total particle density $N$ in the region with velocity as $p=N k T$. In the cases when the atmosphere is not stationary the stationary form of the momentum equation can be less accurate than the hydrostatic equilibrium equation. We have therefore the following possibilities to overcome this difficulty: 1) to use hydrostatic equilibrium equation, 2) to use the stationary form of the momentum equation or 3 ) to use the density as the input parameter from hydrodynamic simulations.

\subsection{Preconditioned ESE and constraint equations}

Substituting for $I_{\mu \nu}$ in (7) from Eq. (12), we obtain an iterative system of non-linear equations for the new populations $n_{l}$. The preconditioning strategy assures this system to be linear in $n_{l}$ provided we know a priori $n_{\mathrm{e}}$. We use here preconditioning within the same transition only (see Rybicky \& Hummer 1992).

ESE are closed using the charge conservation equation

$\sum_{l} q_{l} n_{l}-n_{\mathrm{e}}=0$

where $q_{l}$ is the charge of the ion the level $l$ belongs to.

The electron density is related by particle conservation equation

$\sum_{l} n_{l}+n_{\mathrm{e}}-N=0$

Using the state equation $p=N k T$ and defining $\bar{\mu}$ to be the mean nucleon mass, we can rewrite Eq. (13) as

$-n_{\mathrm{e}} \bar{\mu} v^{2}+N\left(k T+\bar{\mu} v^{2}\right)=m g_{\odot}$.

The set of preconditioned ESE, constraint Eqs. (14) and (15), together with momentum balance (16) can be rewritten in a matrix form for the vector of unknown variables $x \equiv\left(n_{1}, \ldots, n_{n \text { level }}, n_{\mathrm{e}}, N\right)$ (nlevel is the total amount of levels) as

A. $x=b$,

where $b \equiv\left(0, \ldots, 0, m g_{\odot}\right)$. Although the preconditioned ESE are linear in level populations $n_{l}$, this system remains non-linear in the unknown variables due to products of electron density with level populations (the matrix $A$ in 
Eq. (17) depends on $n_{\mathrm{e}}$ ). To correctly solve it we followed Heinzel (1995) and linearized system (17)

$$
\begin{aligned}
\left(A_{0}+\Delta A\right) \delta x & =b-A_{0} x^{\dagger} \\
(\Delta A)_{i k} & \equiv \sum_{j} \frac{\partial A_{i j}}{\partial x_{k}} x_{j}^{\dagger} .
\end{aligned}
$$

As the matrix $A$ depends only on the electron density, the non-zero elements of the matrix $\Delta A$ appear only in its last column.

The system of linear algebraic Eqs. (18) is solved for corrections $\delta x$. The new variables are obtained as $x=$ $x^{\dagger}+\delta x$ and iterations proceed until the changes are small enough, i.e. $\max \left(\delta x_{i} / x_{i}^{\dagger}\right) \leq \varepsilon$.

\section{Calculations}

\subsection{Numerical code}

We wrote the numerical code in C-language. The code uses a 12 levels plus continuum hydrogen atom model. All transition, included continua, are treated explicitly, which means that radiative rates of all transitions are computed in each iteration. We assume all lines to be in CRD which is reasonable in flares due to high $n_{\mathrm{e}}$. The code was tested using a five-levels-plus-continuum model of hydrogen for the temperature structures of the quiet solar atmosphere model VAL3C (Vernazza et al. 1981) and solar flare atmosphere models F1, F2 (Machado et al. 1980). We used the approximate formula for the charge conservation equation that includes ionization from other elements.

$n_{\mathrm{e}}=n_{\mathrm{p}}+1.4410^{-4} n_{\mathrm{H}}$.

The differences for hydrogen level populations and electron densities didn't exceed $15 \%$.

\subsection{Models}

To investigate the influence of the macroscopic velocity field on emergent intensities we proceed as follows. We took the temperature structure of the chromospheric flare model F1 and F2 (Machado et al. 1980) and computed the five-level-plus-continuum models of hydrogen with no velocities. The optical depth scale $\tau_{0}^{\mathrm{H} \alpha}$ for the $\mathrm{H} \alpha$ line center was subsequently used to define the velocity structure. We adopt two different approaches to define velocities.

1. A layer moving with a constant velocity. These layer models are described by three parameters: the velocity of the layer $V_{0}$ and the heights of the lower and upper edge of the layer $\tau_{\mathrm{L}}$ and $\tau_{\mathrm{U}}$, respectively.

2. Models with a velocity gradient. The velocity of gradient models has the form

$V\left(\tau_{0}^{\mathrm{H} \alpha}\right) \equiv \frac{2 V_{0}}{1+\tau_{0}^{\mathrm{H} \alpha} / \tau_{\mathrm{g}}}$.

Note that the velocity goes to zero for $\tau_{0}^{\mathrm{H} \alpha} \gg \tau_{\mathrm{g}}$, to $2 V_{0}$ for $\tau_{0}^{\mathrm{H} \alpha} \ll \tau_{\mathrm{g}}$ and is equal $V_{0}$ for $\tau_{0}^{\mathrm{H} \alpha}=\tau_{\mathrm{g}}$. This
Table 1. Velocity parameters for layer and gradient models. The positive values mean downward velocity. The letter means a position of the moving material ( 1 - lower, $\mathrm{m}$ - middle, $\mathrm{u}$ - upper part of the chromosphere) and the number is the velocity parameter $V_{0}$

\begin{tabular}{|c|ccc|}
\hline \multicolumn{4}{|c|}{ layer models } \\
\hline$V_{0}$ & \multicolumn{3}{|c|}{$\left(\tau_{\mathrm{U}}, \tau_{\mathrm{L}}\right)$} \\
$\left(\mathrm{km} \mathrm{s}^{-1}\right)$ & $(0.01,0.1)$ & $(0.1,1.0)$ & $(1.0,10.0)$ \\
\cline { 2 - 4 } 10 & $\mathrm{u} 10$ & $\mathrm{~m} 10$ & 110 \\
30 & $\mathrm{u} 30$ & $\mathrm{~m} 30$ & 130 \\
50 & $\mathrm{u} 50$ & $\mathrm{~m} 50$ & 150 \\
\hline \hline \multicolumn{4}{|c|}{ gradient models } \\
\hline$V_{0}$ & \multicolumn{3}{c}{$\tau_{\mathrm{g}}$} \\
$\left(\mathrm{km} \mathrm{s}^{-1}\right)$ & 0.032 & 0.32 & 3.2 \\
10 & $\mathrm{u} 10$ & $\mathrm{~m} 10$ & 110 \\
30 & $\mathrm{u} 30$ & $\mathrm{~m} 30$ & 130 \\
50 & $\mathrm{u} 50$ & $\mathrm{~m} 50$ & 150 \\
\hline
\end{tabular}

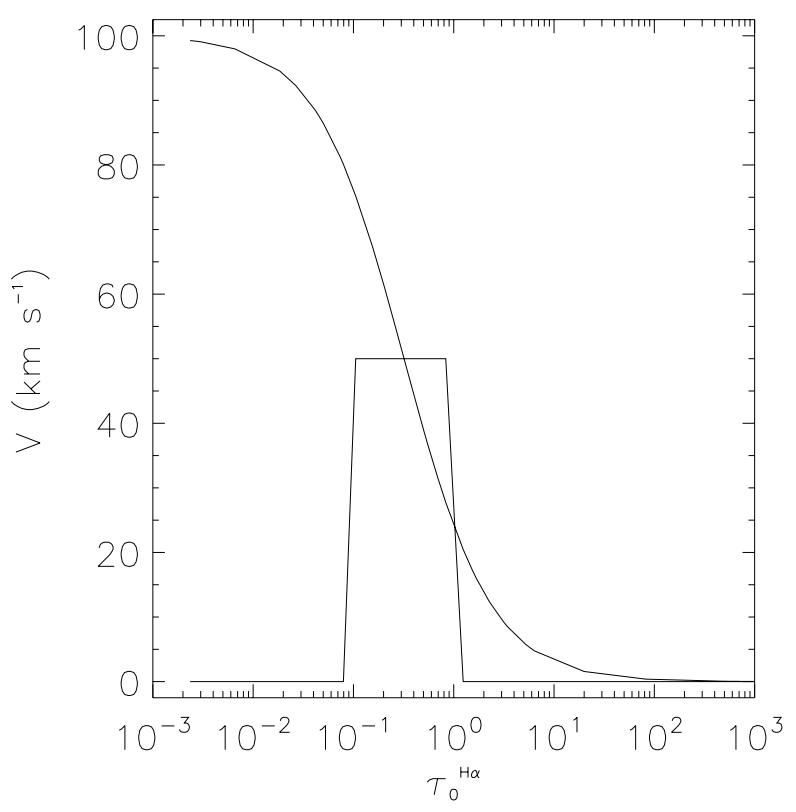

Fig. 1. Velocities of layer and gradient models with the same parameter $V_{0}$ as a function of $\mathrm{H} \alpha$ line center optical depth

formula was used by Mihalas (1976) to describe an expanding atmosphere. Here the formula describes a velocity field with downward velocity increasing with height and we assume that positive values of velocity mean downward motions.

For layer models we chose three regions where we let the material move. These are: the upper part of the flare chromosphere with $\tau_{0}^{\mathrm{H} \alpha} \in(0.01,0.1)$, the middle part with $\tau_{0}^{\mathrm{H} \alpha} \in(0.1,1.0)$ and the lower part with $\tau_{0}^{\mathrm{H} \alpha} \in(1.0,10.0)$. These values of $\tau_{0}^{\mathrm{H} \alpha}$ were substituted for layer-model 

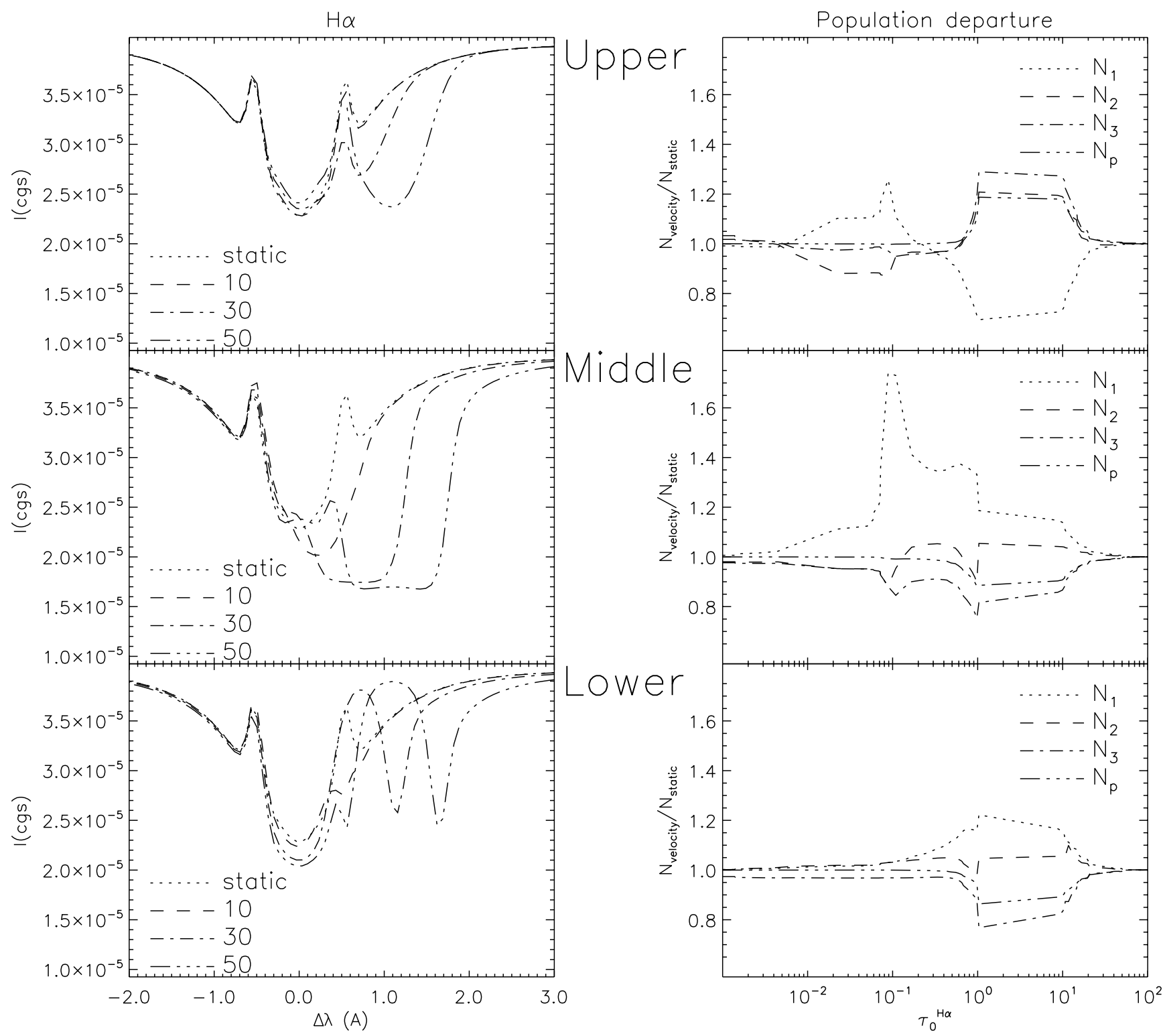

Fig. 2. The $\mathrm{H} \alpha$ line profiles and population departures for layer models with $\mathrm{F} 1$ temperature structure. The population departures are plotted for the models with velocity parameter $V_{0}=50 \mathrm{~km} \mathrm{~s}^{-1}$

parameters $\tau_{\mathrm{L}}$ and $\tau_{\mathrm{U}}$. The gradient model parameter $\tau_{\mathrm{g}}$, which corresponds to the height where the velocity is equal to $V_{0}$, was set to be in the center of each region of the layer models with respect to the logarithmic scale. So the value of $\tau_{\mathrm{g}}$ was $0.032,0.32$ and 3.2 , respectively. To summarize, we have chosen three regions for both types of models denoted upper, middle and lower, with a uniform velocity for layer models and with a velocity increase with height for gradient models.

Once having defined the position of the moving material we chose the velocity $V_{0}$ to be 10,30 and $50 \mathrm{~km} \mathrm{~s}^{-1}$ for both types of models. Note that in the center of each selected region the velocities for both types of models with the same parameter $V_{0}$ are identical. The relationship is shown in Fig. 1.

Values for each particular model are summarized in Table 1.

Hydrodynamic simulations have shown that the density of the moving material is higher than its surrounding (Fisher et al. 1985). As the static form of the momentum equation leads to lower densities in the region with velocities, we used the hydrostatic equilibrium equation instead (see Sect. 2.3 for details).

We started to compute models with the lowest velocity and proceeded to higher velocities. As a starting guess (the level populations and electron density) for models 


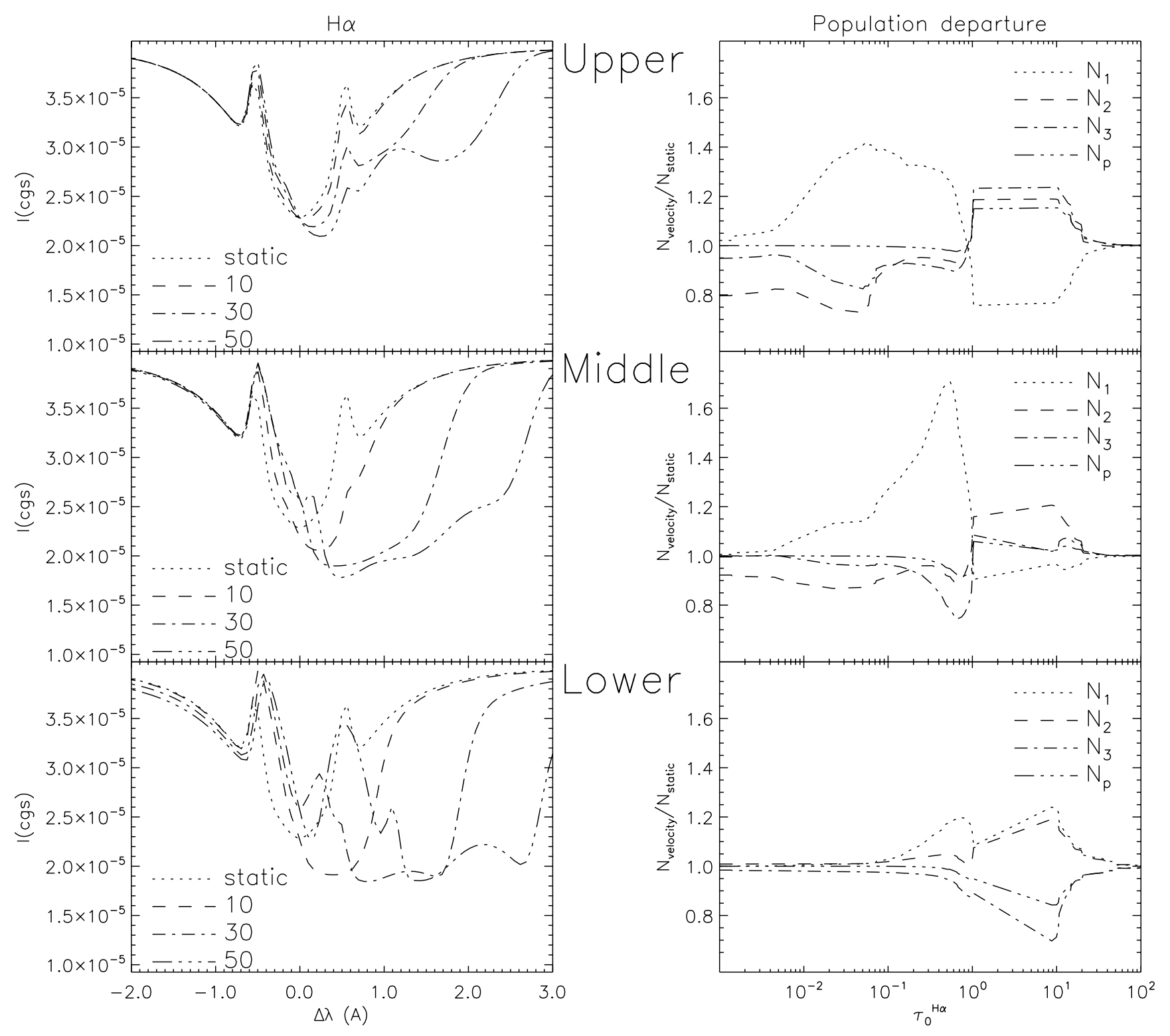

Fig. 3. The $\mathrm{H} \alpha$ line profiles and population departures for gradient models with $\mathrm{F} 1$ temperature structure. The population departures are plotted for the models with velocity parameter $V_{0}=50 \mathrm{~km} \mathrm{~s}^{-1}$

with high velocities we used the results from the models of the same type with a lower velocity. This appeared to be very useful for faster convergence of some models. The convergence (see Sect. 2.4) $\delta n / n^{\dagger}$ below $10^{-4}$ was reached within about 100 iterations for layer models and within about 150 for gradient models.

\section{Results}

The differences between static models and those with velocities are mainly in the emergent intensities. Including the velocities in the NLTE model calculations affects also the level populations as the velocity appears in radiative rates evaluation (Eqs. (7), (8), (9)).

Figures 2, 3, 4 and 5 show the $\mathrm{H} \alpha$ line profiles and departures of the level populations from the static case $N_{\text {velocity }} / N_{\text {static }}$. The lefthand columns contain the profiles for each model together with the static and therefore symmetric profiles. The right-hand columns plot the population departures of the first three levels and protons only for the model with the highest velocity (i.e. for the velocity parameter $V_{0}=50 \mathrm{~km} \mathrm{~s}^{-1}$ ), because the population departures for models with lower velocity have the same structure and only their amplitudes are lower. This indicates that a velocity increase amplifies the population 

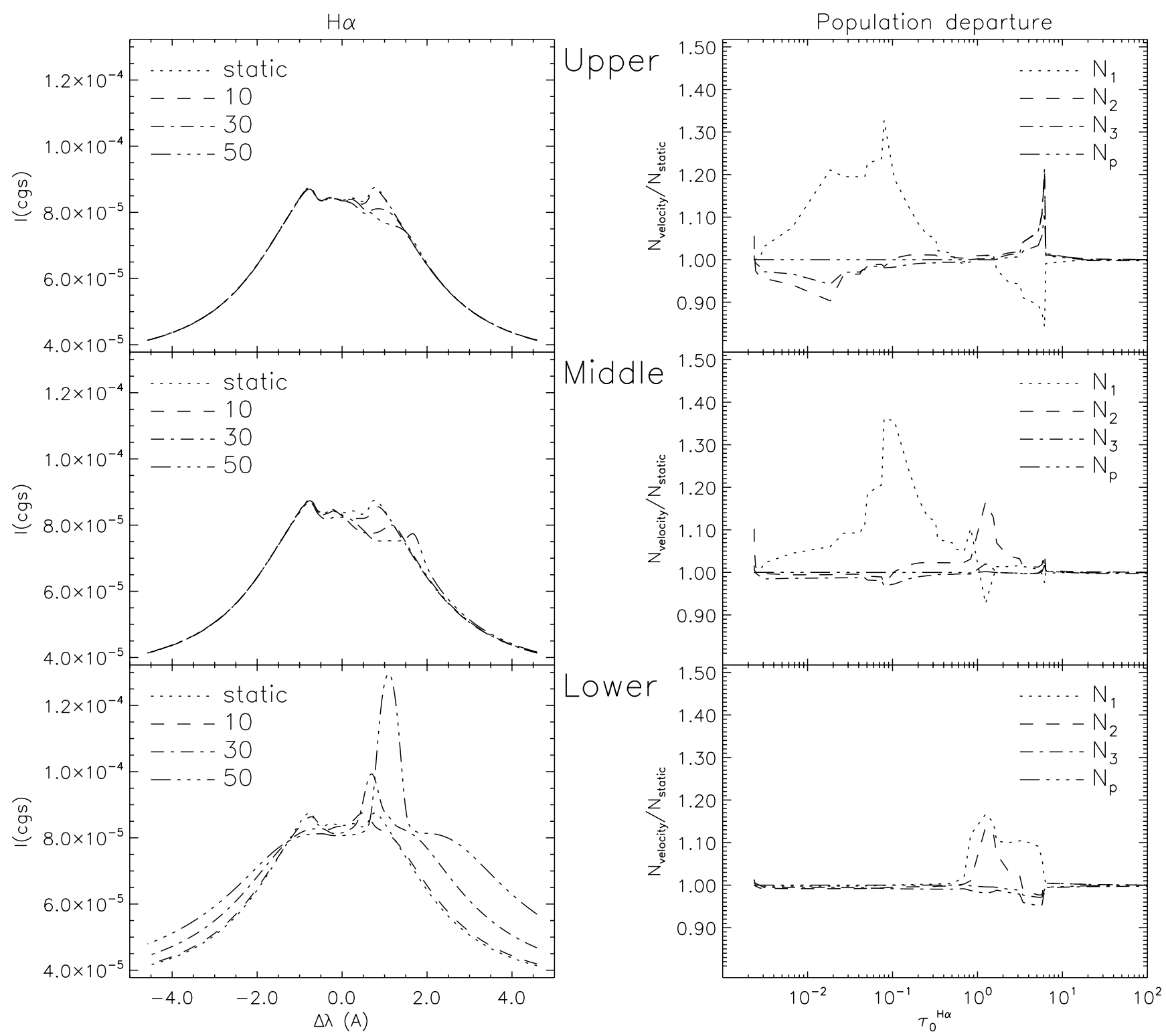

Fig. 4. The $\mathrm{H} \alpha$ line profiles and population departures for layer models with $\mathrm{F} 2$ temperature structure. The population departures are plotted for the models with velocity parameter $V_{0}=50 \mathrm{~km} \mathrm{~s}^{-1}$

departures from static case without changing their height structure. For models with velocities $\approx 10 \mathrm{~km} \mathrm{~s}^{-1}$, which is approximately the velocity of thermal motions, the differences rarely exceed $5 \%$, maximum $10 \%$. To reproduce an observed asymmetry for velocities that are close to the thermal velocity, one can use the static model and perform the formal solution of radiative transfer equation with line absorption shifts caused by the macroscopic velocity. Figure 6 shows the $\mathrm{H} \alpha$ line for $\mathrm{F} 1$ gradient models $\mathrm{m} 10$ and m50. While for the model $\mathrm{m} 10$ the emergent $\mathrm{H} \alpha$ line intensities computed using static population and using the right ones are identical, those for model m50 are different.
For almost all computed models the level populations depart from the static case in two regions. Only the models with non-zero velocities in lower heights have these two regions identical. The first region corresponds to the height of the moving material and therefore depends on the model, while the second one is model-independent and lies in the heights with $\tau_{0}^{\mathrm{H} \alpha} \simeq(1,10)$. The common feature of all models is an overpopulation of the first level and under-population of the second and third level in the moving material.

The collisional rates are the same for the static model as well as for models with velocities as their temperature structure is identical. Thus all differences are due 


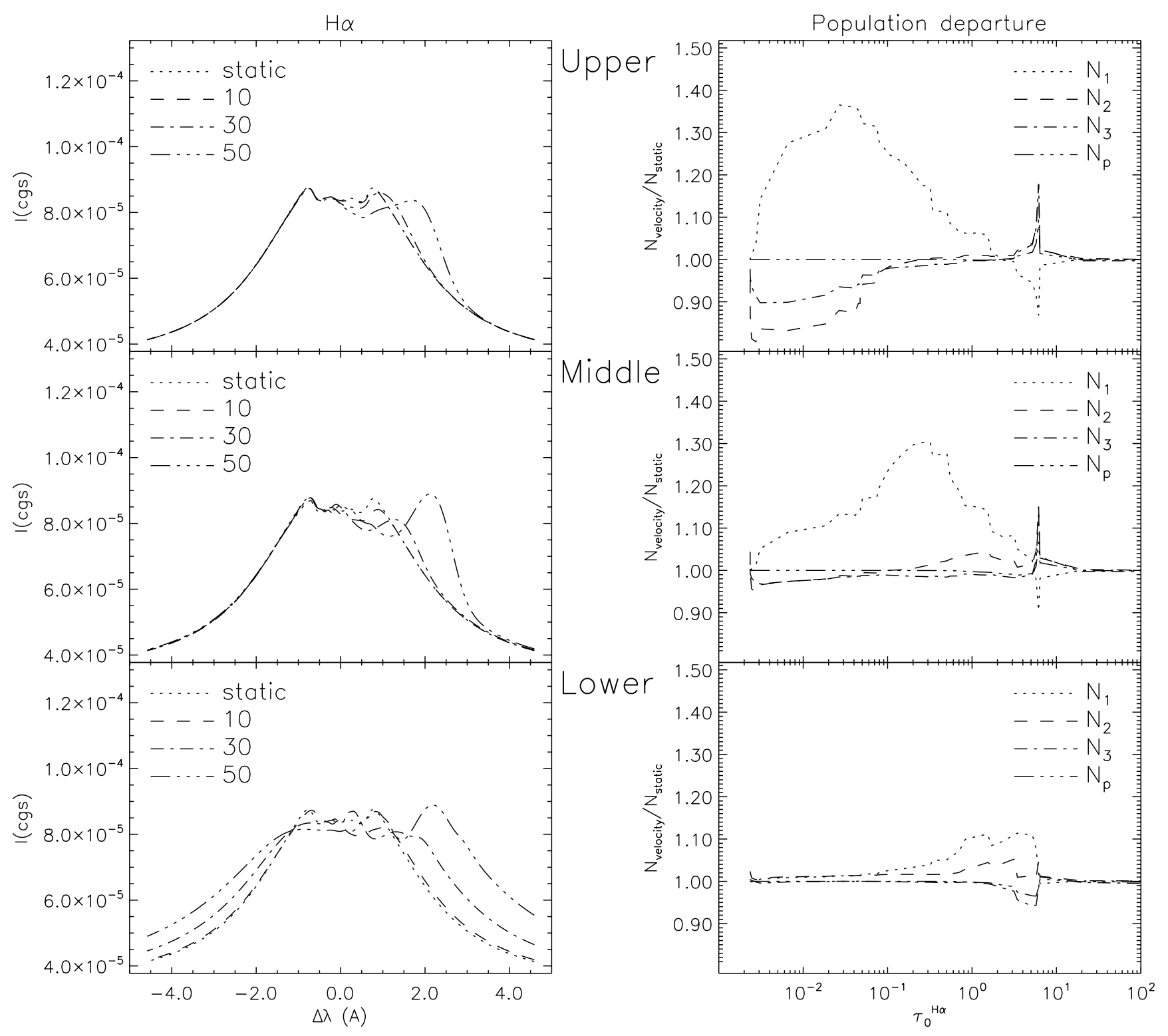

Fig. 5. The $\mathrm{H} \alpha$ line profiles and population departures for gradient models with $\mathrm{F} 2$ temperature structure. The population departures are plotted for the models with velocity parameter $V_{0}=50 \mathrm{~km} \mathrm{~s}^{-1}$

to changes in the radiative rates caused by the shift of the absorption coefficient in the moving material. To find out the changes in the radiation field we selected the layer model in the upper part of the chromosphere with velocity parameter $V_{0}=50 \mathrm{~km} \mathrm{~s}^{-1}$ and $\mathrm{F} 1$ temperature structure, where these two regions are clearly seen. For this model we computed the specific intensity in the $\mathrm{L} \alpha, \mathrm{L} \beta$ and $\mathrm{H} \alpha$ lines for all depths and for upward and downward directions (i.e. for $\mu=1$ and -1 ) and divided it by the corresponding intensity from the static model. Obtained intensity departures (Fig. 7) show that incoming radiation $(\mu=-1)$ plays a crucial role in the atmosphere. In the moving layer $\left(\tau_{0}^{\mathrm{H} \alpha} \in\left(10^{-2}, 10^{-1}\right)\right.$, the $\mathrm{H} \alpha$ wing-line intensity increases by a factor of $10^{4}$, while in the wing of the Lyman lines it is $10^{3}$ times. This depopulates the second level (with respect to the static case) and, via $\mathrm{L} \beta$, overpopulates first level. The incoming line intensities look different in the middle region. The wing-intensity of the $\mathrm{L} \beta$ line exhibits the largest increase, 200 times, in the $\mathrm{H} \alpha$ line, 100 times, while the intensity in $\mathrm{L} \alpha$ increases only 10 times. This force electrons leave the first level and overpopulate the third level. To summarize, the changes of the populations are connected with the increase of the lines intensities. The amount of these changes depends on the ratio of the intensity increases in different lines. If the increase dominates for the resonance lines, the ground 
level becomes underpopulated and the upper levels overpopulated. If the intensity increase of subordinate lines is stronger, under-population appears in the upper levels while the ground level is now overpopulated.

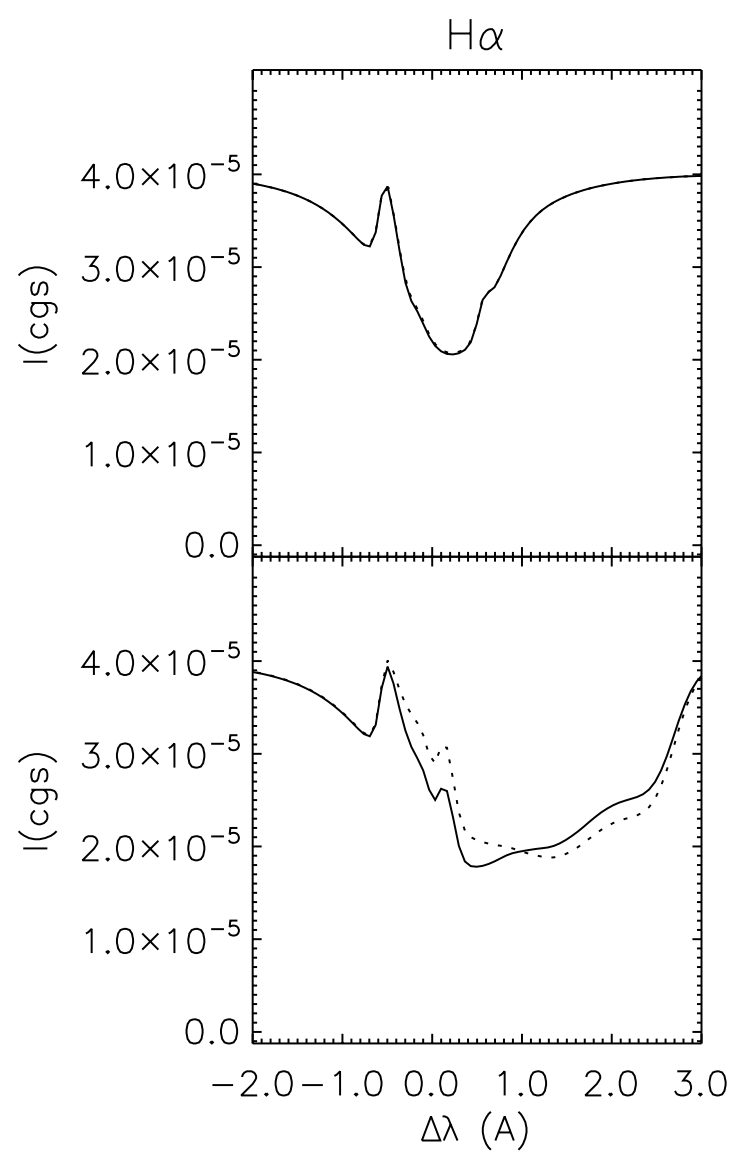

Fig. 6. $\mathrm{H} \alpha$ line computed using static (dotted line) and dynamic (solid line) populations for F1 gradient m10 (upper) and m50 (lower) models

Line asymmetries arise as a result of changes in the optical depth scale for different line frequencies due to shift of the line absorption coefficient. The emergent intensity is approximately equal to the source function at optical depth $\tau \simeq 1$. From the computations we performed it follows that the shift of the line absorption coefficient in the moving material causes a substantial increase of the opacity in the line wing. Height corresponding to $\tau=$ 1 moves up to heights with a different source function. The value of the source function determines whether the emergent intensity will be higher or lower than for the static case.

It should be noted that the line intensity in the blue wing does not differ from the static case because the optical depth scale for the blue wing frequencies remains unaffected. This leads us to the conclusion that if the ve- locities in the flare atmosphere have downward direction, the moving material influences only the red wing of the lines. The same statement holds for upward velocities and the blue wing. Whether the line asymmetry will be blue or red depends on the run of the source function in the atmosphere.

Figure 8 shows the run of the outgoing line intensity with height for some models. The asymmetry of the outgoing line intensity always arises at heights where the velocities become significant, i.e. comparable to the thermal velocity. For layer models it is in the moving layer where the line asymmetry increases and reaches its maximum at the top of the layer. The line asymmetry for the gradient models increases with height as the velocity increases and reaches its maximum at the top of the atmosphere.

\subsection{F1 models}

The source function of $\mathrm{H} \alpha$ for static $\mathrm{F} 1$ model decreases with height. The asymmetric $\mathrm{H} \alpha$ line profiles of F1 models with velocities show the blue asymmetry because the redwing intensity is lowered. In F1 layer models, the moving region appears in the $\mathrm{H} \alpha$ profile as a self-absorption dip with central wavelength corresponding to the velocity in the layer. For F1 gradient models $\mathrm{H} \alpha$ red wing exhibits an intensity decrease that extends from the line center up to the wavelength corresponding to the maximum velocity of the material on top of the atmosphere.

\subsection{F2 models}

The source function of $\mathrm{H} \alpha$ line for static F2 models increases with depth and falls down at the top of the atmosphere. $\mathrm{H} \alpha$ blue asymmetry for $\mathrm{F} 2$ upper and middle layer models is caused by the shift of the optical depth $\tau \simeq 1$ towards the heights where the source function drops. The same mechanism holds for $\mathrm{H} \alpha$ line $\mathrm{F} 2$ gradient models with velocity parameter $V_{0} 10$ and $30 \mathrm{~km} \mathrm{~s}^{-1}$. H $\alpha$ for F2 gradient models with velocity parameter $V_{0} 50 \mathrm{~km} \mathrm{~s}^{-1} \mathrm{ex}-$ hibits a red asymmetry because the optical depth $\tau \simeq 1$ for wavelengths $\lambda \sim 2 \AA$ moves up where the source function is larger than in the static case.

\section{Conclusions}

From our numerical simulations we draw the following important conclusions:

1. The presence of velocity fields affects the level populations via the increase of the downward radiation. Nevertheless, for velocities that do not exceed the thermal velocity of plasma, one can use the static populations for the formal solution of radiative transfer equation including the velocity to reproduce the observed line asymmetries.

2. The line asymmetry is caused by the changes of the optical depth scale for different line frequencies. The 

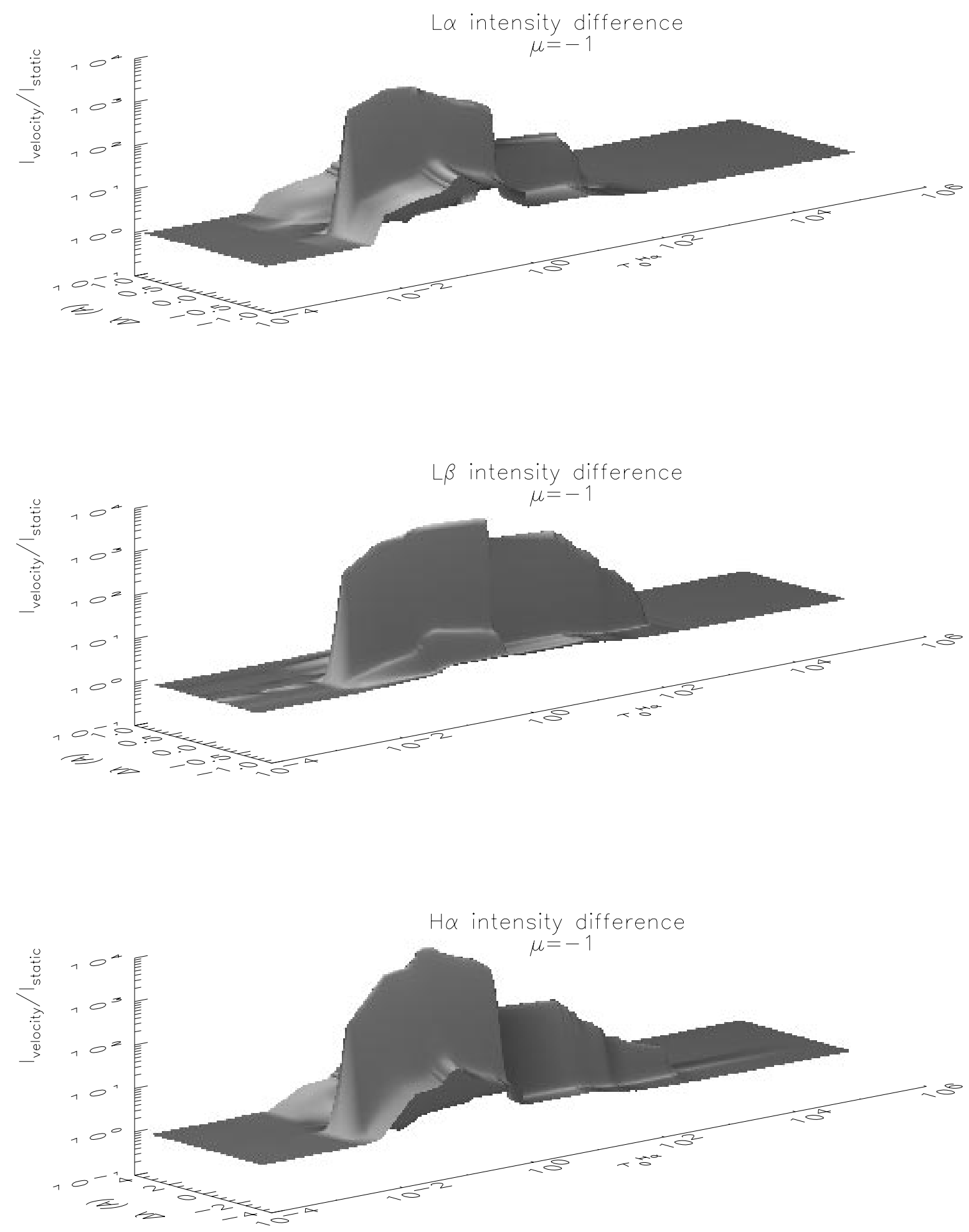

Fig. 7. $\mathrm{L} \alpha, \mathrm{L} \beta$ and $\mathrm{H} \alpha$ line intensity departures for incoming radiation for $\mathrm{F} 1$ layer $\mathrm{u} 50$ model 

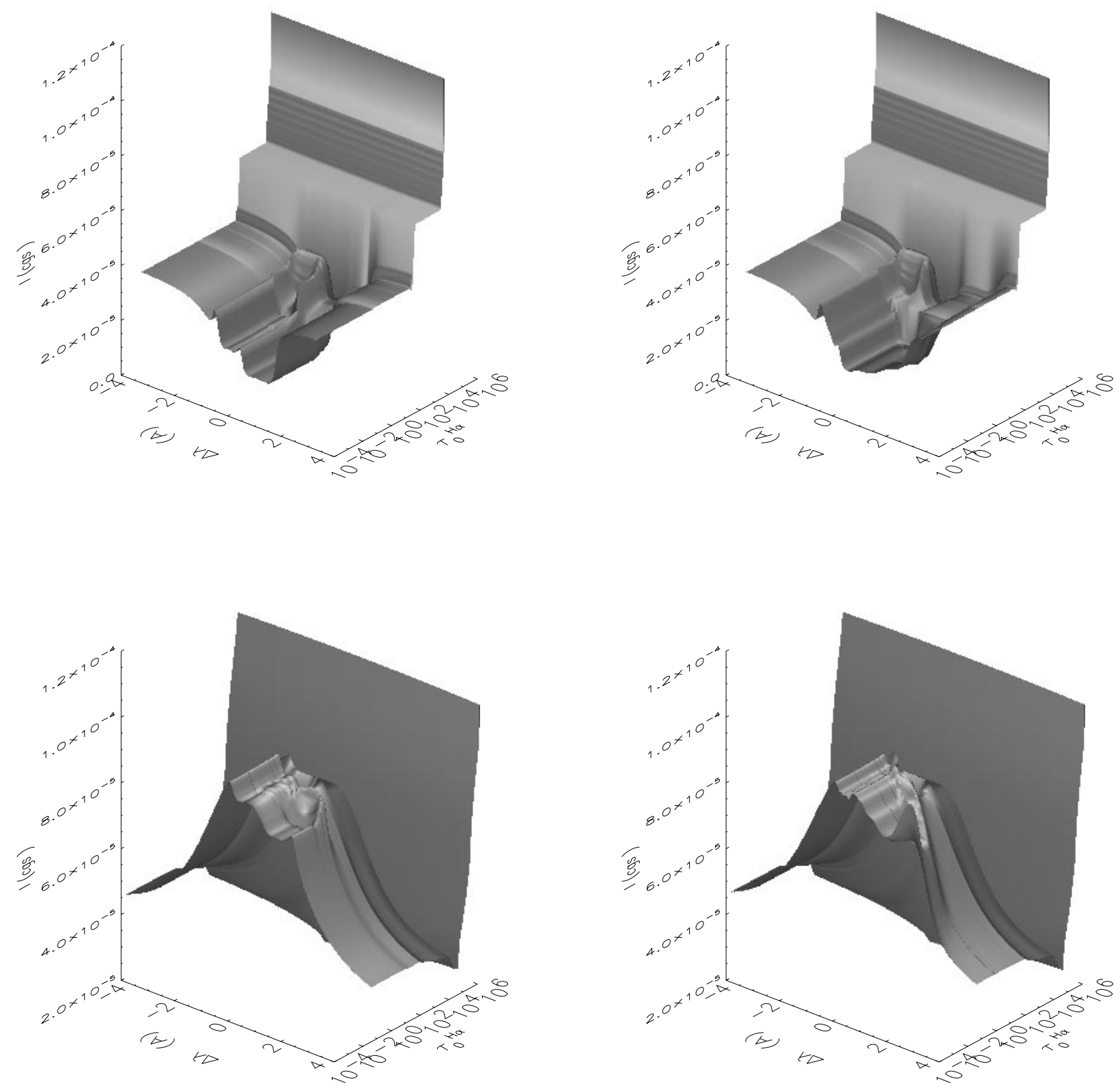

Fig. 8. The $\mathrm{H} \alpha$ line intensity with outward direction $(\mu=1)$ as a function of the optical depth for F1 layer m50 (upper left), F1 gradient m50 (upper right), F2 layer m50 (lower left) and F2 gradient m50 (lower right) models

type of asymmetry depends on values of the source function at heights corresponding to $\tau_{\nu} \simeq 1$.

3. A monotonous velocity field affects only one wing of the line profile, the red one for downward motions, the blue one for upward motions.

4. Application of the bisector method would lead in some cases to reverse velocity, in others to underestimation of the velocity. It includes, besides the part of the pro- file directly affected by the moving material, also a "static" part of the profile. To use the bisector in terms of Doppler shift the static part should be somehow eliminated.

To deduce velocities from asymmetric line profiles using these conclusions, we need to guess how the static profile would look like. For this purpose one could use a symmetric profile from a close observing time or position, 
but then it seems to be necessary to perform NLTE calculations for each particular case.

The determination of the velocity height structure from a single line is inaccurate as an emergent line intensity is affected only in heights where $\tau_{\nu} \simeq 1$, which cover only a small part of the atmosphere for a single line. We therefore need simultaneous spectral observations of more lines, preferably those that originate in different heights, to reconstruct the velocity structure in a flare atmosphere. The more lines will be included the more precise the determination of the height structure of the velocity will be.

Acknowledgements. The author is indebted to Dr. Petr Heinzel for helpful discussions and comments. This work was supported by the Grants No. 205/94/1577 and No. 1199 of the Grant Agency of Czech Republic and by the Grant No. K1-003-601 of the Academy of Science of Czech Republic.

\section{References}

Canfield R.C., Kipplinger A.L., Penn M.J., Wülser J.-P., 1990, ApJ 363, 318

Fisher G.H., Canfield R.C., McClymont A.N., 1985, ApJ 289, 414-425-434
Gan W.Q., Rieger E., Fang C., 1993, ApJ 416, 886

Heinzel P., 1995, A\&A 299, 563

Heinzel P., et al., 1994, Solar Phys.

Ichimoto K., Kurokawa H., 1984, Solar Phys. 93, 105

Machado M.E., Avrett E.H., Vernazza J.E., Noyes R.W., 1980, ApJ 242, 336

Mihalas D., 1987, Stellar Atmospheres

Olson G.L., Auer L., Buchler J.R., 1986, J. Quant. Spectrosc. Radiative Transfer 35, 431

Rybicki G.B., Hummer D.G., 1991, A\&A 245, 171 (Paper I)

Rybicki G.B., Hummer D.G., 1992, A\&A 262, 209 (Paper II)

Somov B.V., Sermutina B.J., Spector A.R., 1982, Solar Phys. 81,281

Švestka Z., 1976, Solar Flares

Švestka Z., Kopecký M., Bláha M., 1962, Bull. Astron. Inst. Czech 13, 37

Vernazza J.E., Avrett E.H., Loeser R., 1981, ApJS 45, 635

Wülser J.-P., 1987, Sol. Phys. 114, 115

Wülser J.-P., Marti H., 1989, ApJ 341, 1088

Zarro D.M., Canfield R.C., 1989, ApJ 338, L33

Zarro D.M., Canfield R.C., Strong K.T., Metcalf T.R., 1988, ApJ 324, 582 